\title{
The Virtual Printshop: A case study on using Virtual Reality in early stages of user-centered product design
}

\author{
Jos Thalen and Mascha van der Voort
}

\begin{abstract}
In the early stages of a product development process (PDP), VR can facilitate communication between designers and product end-users to improve the quality of feedback that users provide to designers. While various forms of VR can already be found in the PDP, they primarily target designers, rather than designers and end-users. Furthermore, available tools and toolkits do not match the skills and requirements of designers in early stages of the PDP. The current paper presents an approach that first determines how to effectively support early stage design activities (referred to as the application) and subsequently provides designers with tools to realize this application themselves. The approach is implemented in an industrial case study involving practitioners from a multinational manufacturer of printing solutions for professional markets. The Virtual Printshop resulting from this case study provides an evaluation platform for various types of early stage product evaluations. A concluding generalization of the cases study results shows that the application can be translated to several other design domains. It was found that there are similarities in how these different design domains integrate $V R$ design tools with their existing tool chains.
\end{abstract}

Index Terms-application, user centered design, methodology, case study.

\section{INTRODUCTION}

$\mathbf{P}$ RODUCT development is a complex matter. An average Product Development Process (PDP) involves market research, concept development, detailed design and engineering, manufacturing, market release and after-sales activities. Throughout these phases a product evolves from an initial concept (a market insight, a first sketch or idea) to a (physical) realization of the product. A challenge in particular inherent to the early phases of the PDP is the lack of concrete design information. Design information (e.g. product dimensions, cost estimations or user requirements) is either not yet available or scattered amongst stakeholders in a multidisciplinary design team. This lack of information forces designers to make decisions based on scarce, scattered or unreliable information [9][16], potentially leading to either unsuccessful products or expensive modifications in later stages of the PDP.

User Centered Design advocates the involvement of product end-users throughout the PDP. End-user involvement can improve the information quality and quantity. End-user feedback for instance facilitates concept generation and selection, or identifies usability issues in an early stage [13][12]. However,

J. Thalen (j.p.thalen@utwente.nl, corresponding author) and M. van der Voort (m.c.vandervoort@utwente.nl) are with the Department of Design, Production and Management, University of Twente, P.O. Box 217, 7500 AE Enschede, The Netherlands with only limited design information available it is difficult to provide end-users with a clear presentation of a product concept and future use context. While traditional boundary objects (means to transfer knowledge between communication partners [3]) such as sketches and mockups properly represent aspects such as style, dimensions or shapes, they can not be used to demonstrate more complex interactive behavior without requiring end-users to interpret technical drawings. Virtual Reality $(V R)$ can extend the collection of early stage prototyping tools by allowing end-users to not only see the future product (which could also be achieved with a concept sketch or mockup), but also experience the product and the interactions within its use context.

The current paper investigates the deployment of VR as a means to facilitate communication between designers and end-users in the early stages of a PDP. As design activities in the early stages of design are different from those found in engineering or manufacturing stages, an approach for identifying early stage design tasks that require support in the communication between designers and end-users. Such an approach is introduced in section III and implemented in an industrial case study, presented in section IV. Section V reflects on the case study as well as the research approach. The paper concludes with an outlook on a more elaborate framework of which this case study is part of.

\section{BACKGROUND}

VR technologies gradually found their way into the realms of the PDP. In the early '90s VR mostly acted as a layer on top of well established CAD systems [17] for visualisation (e.g. CAVE systems and head mounted displays), and, later, as natural and immersive interfaces for existing CAD systems, such as the VRAx immersive modeling system, the NavIMode CAD interface and the ConstructTool immersive modeling system, all of which are described in [24]. The substantial costs and a focus on large and complex data sets made VR primarily applicable in larger industries such as aerospace and automotive design. Advancements in hardware and software have reduced costs and extended the application scope of VR to simulation, training, prototyping and evaluation purposes [11].

These product design applications generally exploit the ability of VR to allow non-existing products or environments to be experienced in a natural and realistic way. This is beneficial when the real world situation is too dangerous (e.g. 
a drive simulator, as described by Tideman [20], when an environment needs to be controlled (e.g. in simulation and evaluation as described in [14]) or when physical prototyping is too expensive or simply not possible yet (e.g. virtual prototyping, as described in [4]). The examples of advantageous applications of VR technologies in the PDP illustrate the substantial set of applications available for the field of product design. However, the majority of these applications aim to support collaboration between designers rather than for instance between designers and end-users. Furthermore, applications generally target advanced stages of the PDP, such as engineering and manufacturing. As we are interested in facilitating communication between designers and end-users in the early stages of the PDP, additional preliminary research was conducted to assess the current state of VR in this area.

\section{A. VR in Early Stages of Design}

A series of in-depth interviews with over 40 designers, engineers and managers from four multinational companies (involved in automotive design, mechatronic design, mechanical engineering and consumer electronics) showed that the use of VR was mostly limited to the use of CAD systems and $3 \mathrm{D}$ displays for engineering reviews. In the early stages of product development, designers sometimes used simplified CAD models for quick visualizations, but did not involve VR technologies otherwise. Nevertheless, interviewees acknowledged the potential benefits of applying VR in early stages of the PDP, after outlining possible applications. It was however difficult for the participants to translate the theoretic VR applications into concrete requirements. The same finding was reported in [7], where participants "had difficulty expressing and developing ideas for specific applications of a technology they had little experience of". In a subsequent VR demonstration session participants were shown various forms of VR technologies being used in design applications, including an augmented reality factory layout application, an immersive drive simulator, a 3D virtual usability test lab and a 3D interactive experience lab [19]. The participants were now able to see and discuss various interpretations of how VR could be used in the PDP. The session ended with a group discussion about how to actually realize these applications. Participants pointed out that in the early stages of development, it is important to be able to work quickly, as creativity can not easily be predicted or guided. It was found to be important for VR design tools to be operated directly by designers, i.e. realizing the VR application themselves instead of being facilitated by other departments or an external company. Furthermore, designers are fond of their tools and very skilled in using them. Ideally, VR design tools should therefore fit existing tool chains rather than replace them.

The preliminary research shows that designers recognize the potential benefits of using VR in the early stages of the PDP, but apparently lack the tools (or awareness of tools) that enable them to realize these applications themselves.

\section{B. Problem Definition}

To better understand this mismatch between applications and tools to realize those from the perspective of a product designer, an analogy with an established design tool, Adobe Photoshop, can be made. Photoshop is a tool that is used by the designer to create visualizations that help with showing a product concept to customers. The use of the visualization is referred to as the application of, in this case, graphics software. Designers know what kind of applications they can realize with the tool, and (by training or experience) know how to use the tool to do so. When this analogy is translated back to VR, two issues emerge. Firstly, the majority of existing VR tools originates from research in computer science, and often consists of toolkits that extend programming languages with VR specific functions (see [25] for an extensive survey). Examples include VR Juggler [5], OpenTracker [18] and ARToolkit [10]. While these toolkits provide a good platform for further development by experts, they are by no means usable by non-expert designers; in the analogy of Photoshop, it would be like providing designers with a programming language and GUI libraries to create their own Photoshop. Secondly, user friendly tools such as ComposAR [23] or DART [15] do provide a more accessible authoring tool but reduce the tool's flexibility (e.g. the range of applications). More flexible VR development suites, such as Autodesk's Showcase, VRED Professional or Dassault Systemes' 3DVIA primarily target later stages of the PDP such as engineering and simulation.

The approach presented in the next section will address the gap between the potential benefits of VR applications in early stages of a user centered PDP and the tools available for designers to realize these applications.

\section{APPROACH}

The research approach is characterized by its participative and hands-on nature, meaning that design practitioners are actively involved in the identification of VR tool requirements and the evaluation of application and tool prototypes. The approach involves five major phases.

1) Application Definition - The first step in the approach is for the researcher and the practitioners collaboratively define the advantageous application of VR within the design process of the participating company. Collaboration between the researcher and practitioners is required to exchange in-depth knowledge of the respective fields of expertise.

2) Application Development - Given the application outline, a functional prototype of the application is developed by the researcher to allow practitioners to experience and evaluate it. Throughout this development, practitioners will be involved to test and refine the application, making sure that the prototype matches the envisioned VR application.

3) Application Review - The functional prototype is used by the practitioners to verify the effectiveness of the VR application. Here the main question is whether or not the use of VR indeed facilitates the intended design activity (as defined in step 1).

4) Tool Selection - After verifying the application the researcher provides the participants with VR design tools 
(by selecting, combining or adapting existing tools) that enable designers to realize the envisioned application themselves (up to a desired level of customization). These tools are not necessarily the same tools as used by the researcher to develop the prototype, because the skills and requirements of designers differ from those of the researcher.

5) Generalization - The VR application and the accompanying design tools, which are custom-made for one specific company, are presented to companies from various design domains (e.g. automotive, consumer electronics, etc.) in order to assess the validity of the applications and tools across design domains.

This approach has been implemented in an industrial case study. The detailed proceedings of the case study are presented in the next section.

\section{CAse Study}

The case study was carried out for a multinational manufacturer of printing systems for the professional market. The company's design department is primarily involved with the design of user interfaces and user-product interactions, and includes interaction designers, product designers, visual designers, usability engineers and software prototypers. While the end-users of this product are typically trained printer operators, designing a good user interface is challenging because of the technical complexity of the machines, but also because of the various use contexts in which the products are used (e.g. universities, small offices or professional printshops). Consequently, the design department of this company is interested in finding new tools and methods for actively involving their end-users in the development and evaluation of new printers. The following subsections provide a detailed description of how the five phases of our approach were implemented in this case study.

\section{A. Application Definition}

The early stages of the company's PDP include several activities in which the designers work with end-users. For example, the designers conduct interviews with end-users, do site visits and invite end-users to evaluate new user interface prototypes. To determine which activities can benefit from VR, the researcher and participating designers need to share and exchange domain knowledge effectively. In a group workshop, which was the first major event in the case study, we applied a participatory design technique based on storyboards to facilitate the exchange of knowledge between designers and the researcher. The use of visual storyboards was inspired by various participatory design methods such as Inspiration Cards [8], Pivots [21] and the Future Technology Workshop [22]. In our workshop the designers were asked to describe design activities by arranging individual frames into a coherent story. Each frame visually depicted a generic event, such as 'working on a computer', 'having a meeting' or 'talking to a customer'. The designers were also asked to insert 'technology frames', which depicted a specific VR technology, such as augmented reality, haptic input devices or motion

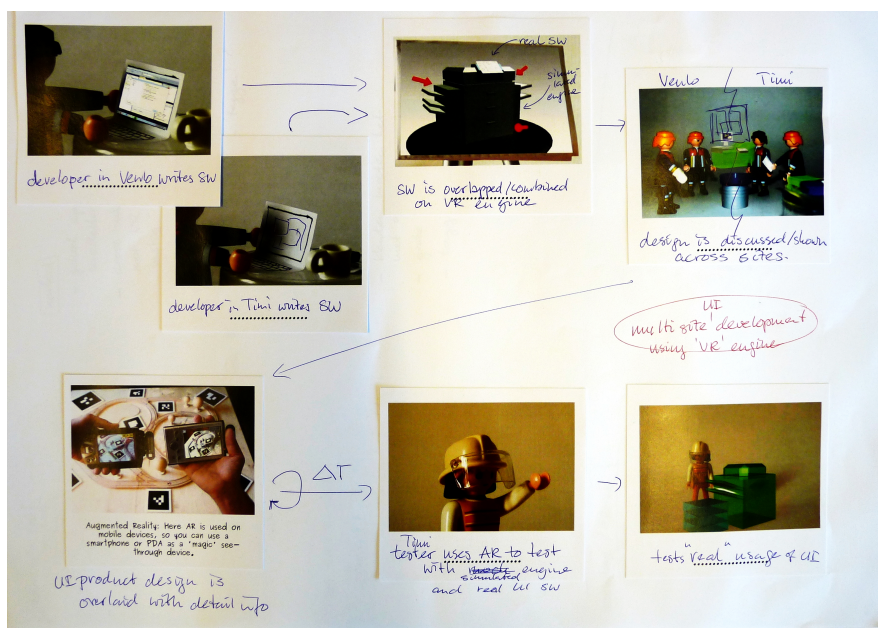

Fig. 1. One of the individual storyboards created during the workshop The storyboards visualise the designer's ideas about how to apply VR in the product development process.

tracking. The participants (the workshop included a total of 12 participants) first created individual storyboards (see figure 1), which were merged into four group storyboards after a round of presentation and discussion. The four resulting group storyboards visualise different situations in the design process where VR applications are considered useful. For instance, one of the storyboards describes the use of augmented reality to allow end-users to see virtual future copiers in their own office. Another storyboard describes the use of VR to support detailed design of machine components by allowing engineers to inspect the future product in virtual reality. The contribution of the storyboard workshop lies not in the novelty of these applications, but rather in giving VR technologies a familiar context, which makes it easier for designers to identify and discuss requirements for the application.

After participating in the workshop, the designers were able to provide the researcher with a clear description of their challenge, as well as an indication of how they expect VR to face that challenge. According to these results, the designers' primary reason for using VR is to improve the experience that end-users have while they are involved in the evaluation of new printer (interaction) concepts; they should feel 'at home' while operating a printer. The use context influences the interaction between the operator and the printer; ambient noise may distract the operator, or the operator maybe involved in other tasks than printing. Given the influence of the use context on the interaction with printers, designers should take the use context into account during the design and evaluation of the user interface and interactions. However, the dedicated usability lab that is currently used for this purpose (see figure 2(a)) does not represent a realistic use context; it is an empty room with a clinical appearance, while a real use context typically consists of crowded printshops where phones are ringing and customers are calling for attention (see figure 2(b)). An envisioned 'virtual use context' is expected to provide a more realistic, flexible (it can be adapted to match the use context of different end-users) and controlled (designers can decide what does or does not happen in the 


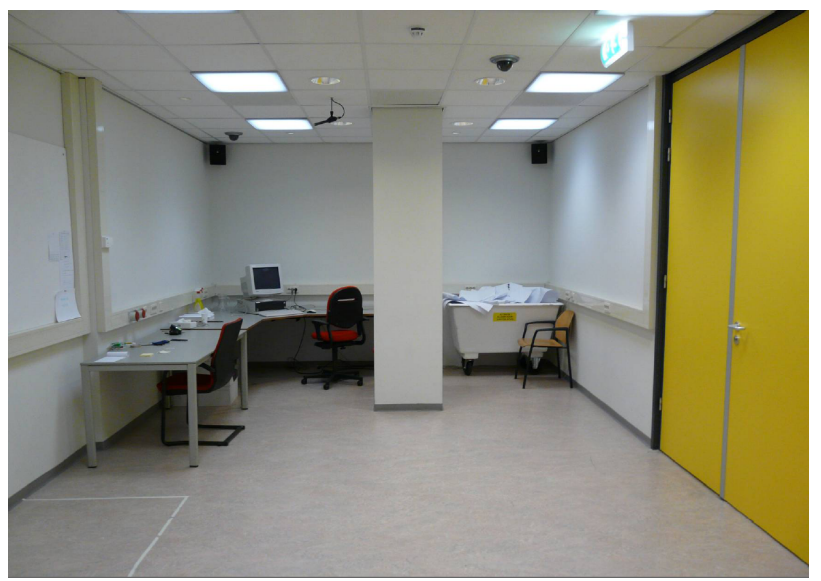

(a) The test room currently used to evaluate new product concepts.

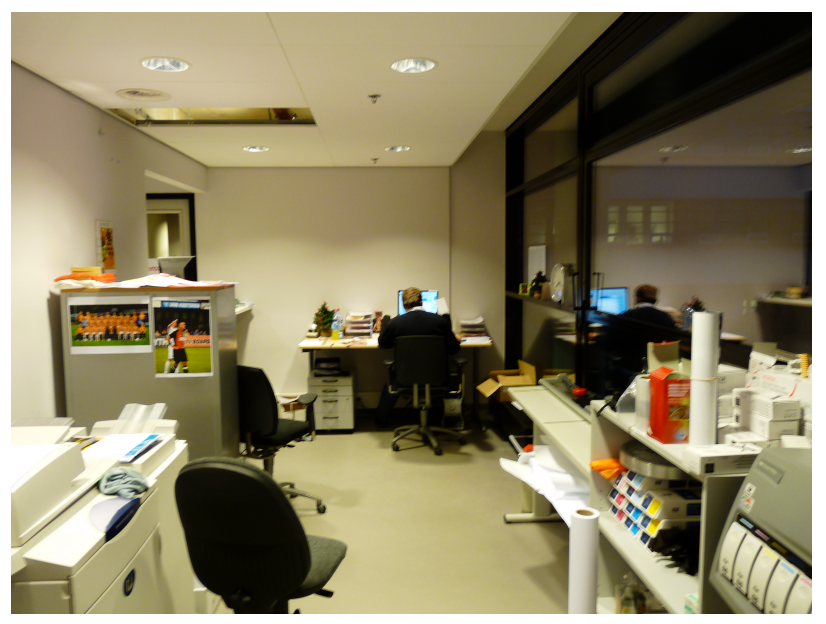

(b) The crowded environment of a typical printshop.

Fig. 2. Participants in product evaluations (e.g. printer operators) should feel 'at home' during a product evaluation, which can not be achieved in the current test lab (figure 2(a)). VR is used to create a more realistic and familiar test environment.

use context) environment for conducting early stage product evaluations.

\section{B. Application Development}

The second part of the case study involves the development of a VR application that provides designers with a virtual use context that can be used for early product evaluations involving end-users. This interactive virtual use context requires an appropriate technical implementation; designers and end-users need to be able to immerse themselves in the environment. Two technical alternatives were discussed with the design practitioners. Mobile augmented reality $(A R)$ could be used to place end-users in the virtual use context and let them act-out work habits and task sequences. Alternatively, a fully virtual environment such as a CAVE or a relatively simple first-person game environment could provide a different experience yet still suit the desired application. In order for design practitioners to assess these technical alternatives, it was decided to develop and evaluate two application directions, namely the 'Virtual Printshop' and the 'Augmented Reality Printshop'.

1) The Virtual Printshop consists of a digital 3D virtual

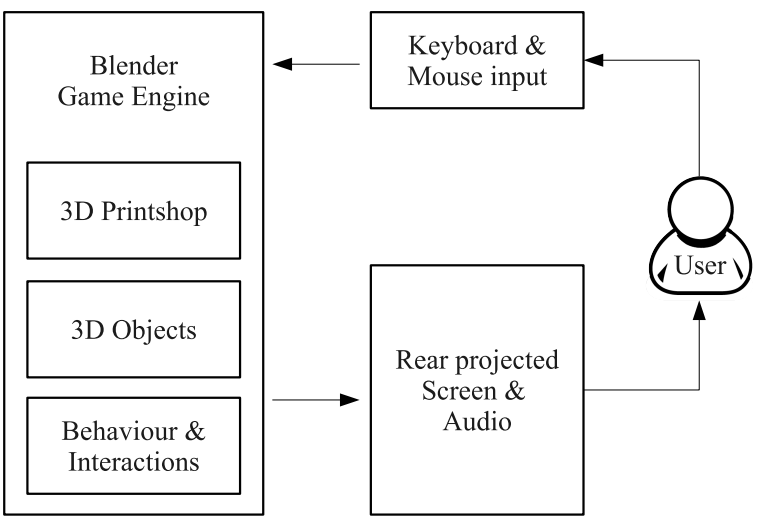

(a) Diagram of the Virtual Printshop setup. The Blender Game Engine is used to model, control and render the virtual environment, projected on a large rear projected screen.

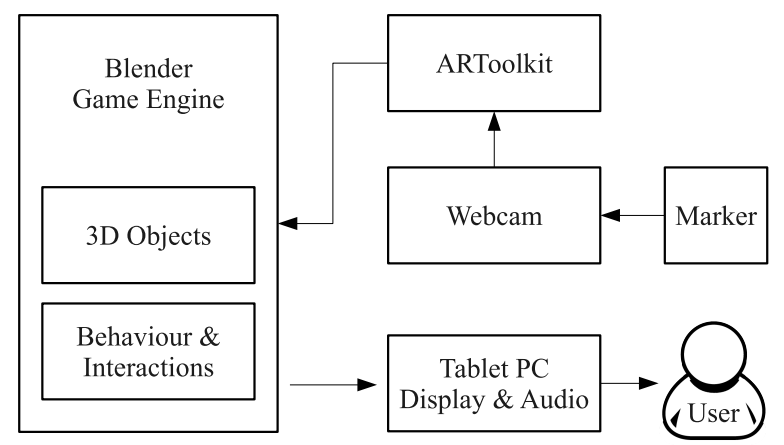

(b) Diagram of the AR Printshop. The Blender Game Engine is connected to ARToolkit, which takes care of marker detection using a webcam on the tablet PC.

Fig. 3. Diagrams outlining the software used in the two virtual printshops.

office that is projected on a large rear-projected screen $(3 \times 2 \mathrm{~m})$. Designers, positioned in front of this screen, use a keyboard and mouse to navigate a first-person perspective through the environment. The application runs on a standard desktop computer and uses the Blender game engine [6] for rendering and controlling the interactive 3D environment (see figure 3(a)).

2) The AR Printshop consists of a tablet PC equipped with a camera. Pointing the tablet on a visual marker will display corresponding 3D models on the tablets display. This allows designers to physically move around while exploring the augmented reality environment, pointing at specific markers. The augmented reality is based on a combination of ARToolkit [10] and the Blender game engine (see figure $3(\mathrm{~b})$ ).

An existing printshop has been used as a reference for creating virtual models of office furniture, machinery, layouts and room decorations (see figure 2(b)) that provide a common basis for both applications. Some of the objects are interactive; the printer models have system states, such as 'printing', 'idle', or 'out of paper' that can be changed by user interactions. A 3D authoring tool [6] was used by the researcher to create the two applications.

In addition to evaluating the difference between the Virtual Printshop and the AR Printshop, we were also interested in the required level of realism of the virtual contexts. Without proper 

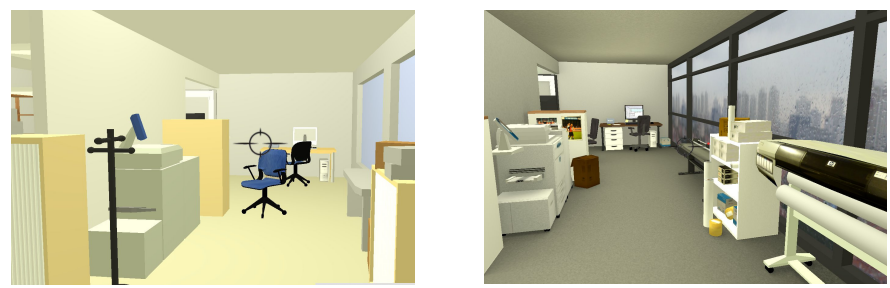

(a) The 'Virtual Printshop' in low level of realism (left) and high level of realism (right).
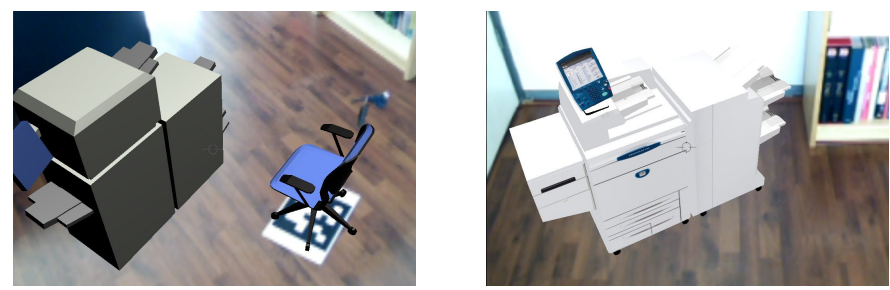

(b) The 'Augmented Reality Printshop' in low level of realism (left) and high level of realism (right).

Fig. 4. The two application prototypes and their levels of realism.

references it is difficult for design practitioners to indicate what level of realism they need for a use context to be effective in product evaluation sessions. Without a sufficient level of realism users may not recognise an environment or objects, or may not take the evaluation task seriously. Creating highly realistic environments on the other hand (visually, but also in terms of audio and interactions) is time consuming and therefore less feasible in the early stages of a design process.

In order to see how the level of realism affects the VR application, both printshop applications were created with two degrees of realism. The high realism applications include visually rich objects (e.g. detailed geometry, photo-realistic textures and realtime shadows), 3D sound and interactive animated models (e.g. moving printer parts). The low realism applications use models with less detailed geometry, no textures, no shadows, regular stereo sound and lack animated objects. Figure 4 illustrates the different levels of realism used in the Virtual Printshop and the AR Printshop.

\section{Application Review}

The AR Printshop and the Virtual Printshop were deployed in a test case. The aim of this test case was to assess the effectiveness of product evaluations in a virtual use context and to compare the Virtual Printshop to the AR Printshop.

A group of four designers from the company was asked to carry out a product evaluation in both the AR Printshop and the Virtual Printshop, and compare these sessions to the product evaluation sessions in the traditional test environment (i.e. a product evaluation in the dedicated usability lab, see figure 2(a)). The topic of the product evaluation consists of a new paper feed tray, for which three design concepts have been created. Each concept represents specific positions and opening mechanisms of the tray. This topic was chosen because it covers physical interactions between the operator and the product (i.e. operators have to be able to reach the tray), as well as interactions between the user interface and the tray (i.e. the user interface should inform operators about

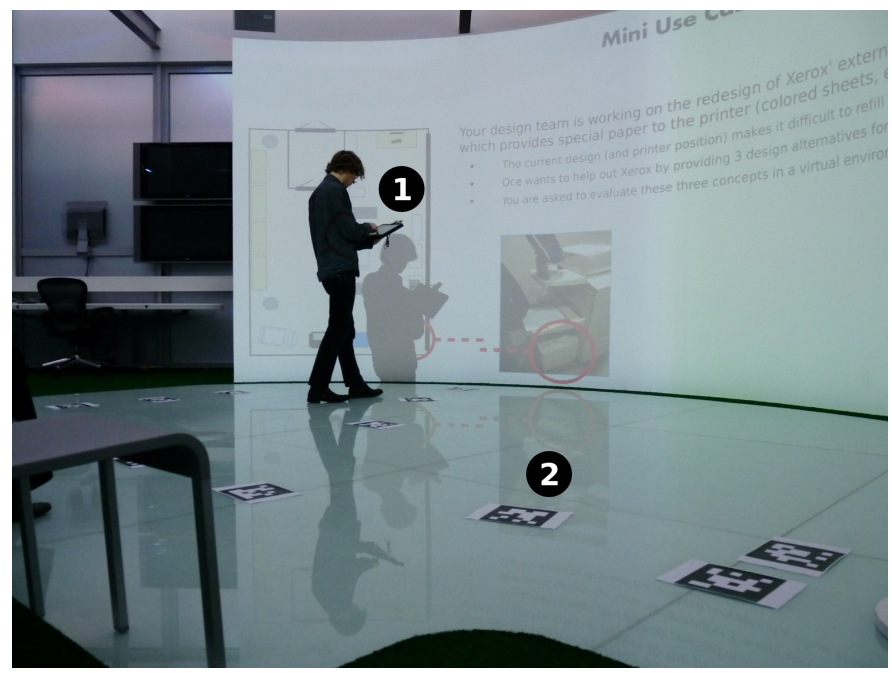

(a) The Augmented Reality Printshop, in which a designer uses an augmented reality tablet (1) to walk around the augmented reality markers (2).

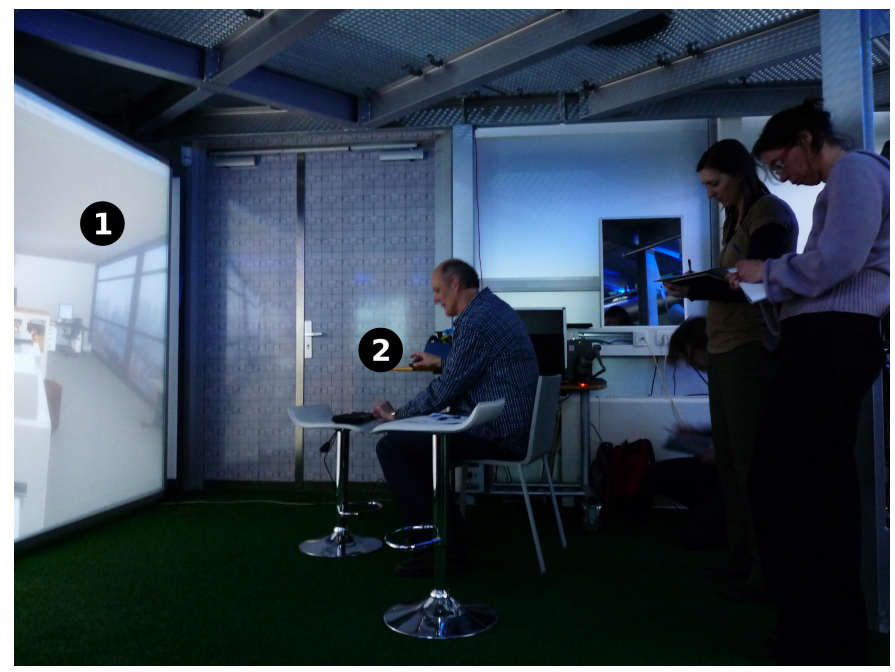

(b) The Virtual Printshop, in which designers (2) operate a first-person perspective 3D environment projected on a large screen (1).

Fig. 5. The two prototypes of the virtual printshop in use during the application review.

an empty paper tray). While the product evaluation session should also include real end-users, it was decided to only involve designers because of the experimental nature of the applications. During the test session, designers who operated the virtual printshop (i.e. control the keyboard and mouse, or hold the AR tablet) temporarily acted as end-users.

The participants were subsequently introduced to 1) the high realism Virtual Printshop, 2) the low realism Virtual Printshop, 3) the high realism AR Printshop and 4) the low realism AR Printshop. The designers spent about thirty minutes in each of these four virtual printshops, carrying out a use scenario to evaluate the different paper tray concepts. The use scenario (which was the same throughout the evaluation session) involves the following steps.

1) A printer runs out of paper and switches to idle

2) The operator collects a new pack of paper

3) The operator opens the tray and inserts new paper

4) The printer resumes its print job 


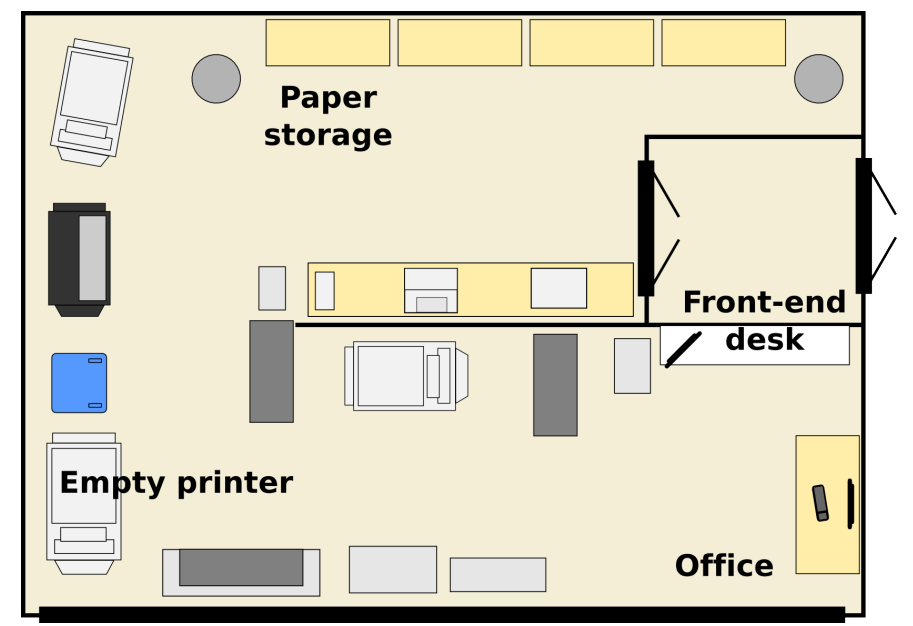

Fig. 6. Layout of the printshop. During the product evaluation, the printer on the lower left runs out of paper and needs to be refilled. The participants collect a new pack of paper from the paper storage. A queue of customers forms at the front-end desk during the evaluation.

Figure 5 shows the group of designers as they carry out the evaluations in the two different virtual printshops. Figure 6 illustrates the key elements of the use scenario in a layout of the printshop. After completing the evaluation sessions a group discussion was held to gather feedback on the different types of virtual environments.

The discussion focused on the differences between the Virtual Printshop and the AR Printshop, and the difference between the two levels of realism of the printshops. Given the low number of participants and the experimental nature of the use case we focused on gathering qualitative rather than quantitative feedback. Consequently, the insights regarding the differences between the Virtual Printshop and the AR Printshop, and the difference between the two levels of realism should be considered valid only within the context of this case study.

Overall, both printshop applications allow users to move from one printer to another, and to include workflow elements such as receiving printing orders from customers, postprocessing a print job or doing administrative tasks on a computer. Being virtual, the workflow can easily be adapted to assess the effects of room layout modifications or changes in machines or personnel. In addition to doing product evaluations and workflow analyses, the environments can also be used for generating and quickly evaluating new ideas or communication purposes (e.g. interactive demonstrations of new products).

With respect to the differences between the Virtual Printshop and the AR Printshop, it was found that designers preferred the Virtual Printshop over the AR Printshop. Designers indicated that the augmented reality approach does not really achieve a feeling of being in the printshop; the restricted view through the tablet computer, the lack of walls and the sudden 'popping up' of objects in the augmented reality environment prevent the participants from staying 'immersed' in the virtual world. A benefit of mixed reality on the other hand is that it also simulates physical interactions; designers had to kneel down in order to reach lower paper trays. However, such physical and ergonomical aspects are more easily tested through wooden or paper mockups, limiting the added value of VR in this area.

It was found that the level of realism of products should be high, comparable to the high-level demo. A printer in the lowrealism printshop triggers less feedback than a highly realistic printer, and it makes it difficult to assess the dimensions of the object. The realism of the context is less important, but should be slightly higher than the low-level demo (e.g. add shadows, visual cues for interaction). Participants agreed that it is a matter of experience to know what to include (or not) in the context (e.g. is a clock a part of the workflow?). In context visualization, the layout adds sufficient reference for recognizing a certain printshop; chairs do not need to be a 1:1 copy of the real chairs, as long as there are chairs on the correct location in the room. Apart from visual realism, participants also noted that sound significantly affects the sense of realism. The low-realism sound (on or off) was considered confusing, even though it provides a clear indication of printer status. It was concluded that sound should be either realistic (stereo, 3D, interactive) or completely left out.

Following these findings, it is concluded that the application facilitates the anticipated design task, namely early stage product evaluations. Designers indicated that the virtual printshops contribute to a more realistic use experience, thus answering the first question of the test case. Furthermore, based on the feedback from the designers it is decided to focus on the realisation of the Virtual Printshop rather than the AR Printshop. It was also found that even with a lower level of detail, participants still recognize a use context, as long as there are sufficient references to the real-life environment.

\section{Tool Selection}

Having established the Virtual Printshop as the application, the next step in the case study is to provide designers with appropriate tools to realize this application themselves. Tool selection depends on several aspects, such as the required level of realism of the resulting virtual environment, the available skills (e.g. modeling or programming the environment) and possibly the integration with other tools used in the PDP (e.g. to use data from existing model repositories). Given their experience with the application prototype earlier in the case study, the designers were able to contribute to the tool selection by expressing concrete requirements and preferences. Designers were introduced to three steps required to realize the Virtual Printshop application, and the range of tools available for each of these steps.

- Geometry Modeling - This step involves the creation or importing of model geometry (including shapes, colors, materials, etc.) needed for the virtual environment. In the virtual printshop this includes printer models, furniture and avatars. Tools available for this step range from regular 3D modeling suites and CAD software, to simply importing existing models from internal or external model repositories.

- Scene Integration - Scene Integration involves the creation of a virtual room or area and putting the 3D 
geometry in this environment. In the virtual printshop the room consists of the printshop room, and the arrangement of printers and furniture within the printshop. Tools available for this step range from 3D modeling suites and CAD software to dedicated interior decoration and layout software.

- Behavior Modeling - The third step involves defining the interactive behavior of objects and the environment. In the virtual printshop this includes the system behavior of printers (e.g. being able to print and output paper) and the ability of avatars to form a queue at the printshop's desk. Tools available for behavior modeling range from regular programming and scripting languages to visual programming languages and pre-programmed behavior.

The researcher explained how different tools used for each of these tasks lead to different levels of realism and virtuality. For instance, higher levels of realism require more complex modeling tools such as game engines, while low realism environments can be created with easy to use offthe-shelf interior design software. Sharing this information with designers enables them to assess the trade-offs between application characteristics and tool requirements, but also allows for a comparison between the tools needed to realize the VR application (the VR tool chain) and the tools already available within the company. Taking this information into account, the participating designers were able to compose a tool chain and allocate tool chain components to specific departments or disciplines.

- Geometry Modeling is allocated to product designers who already work with CAD models. During the prototype session it was found that some objects, such as printers, should have a relatively high level of realism. These models could therefore be directly imported from the company's existing CAD database. Other objects, such as furniture, have lower requirements with respect to realism (or similarity with a real-life environment) and can therefore be imported from generic 3D databases, such as Google 3D Warehouse [1].

- For Scene Integration, designers prefer a low threshold and easy to use tool rather than a more flexible but complex tool such as a generic game engine. Interior decoration software such as SweetHome3D [2] provide a user friendly way to create virtual environments, and allow users to import other 3D assets (e.g. printers and furniture). This part of the tool chain would be used by usability engineers, who are usually in charge of arranging product evaluations.

- The designers indicated that Behaviour Modeling can be allocated to dedicated prototypers (designers trained in creating interactive software prototypes or mockups), who are already available in the design department. Given their experience with software prototyping the Behavior Modeling tools can focus on functionality and flexibility rather than ease of use.

The tool chain was verified in a series of follow-up workshops. Here designers, usability engineers and prototypers were involved in carrying out their respective parts of the tool chain. Designers and usability engineers used SweetHome3D for creating virtual environments (which they had to do based on e.g. a floorplan and photos of a reference environment). Assets (furniture, printers, etc.) were imported from internal CAD databases as well as public databases such as Google 3D Warehouse. The resulting virtual environments were used in a subsequent workshop in which the Blender Game Engine was used for adding behavior to these environments (e.g. ability to walk through the environment, interact with objects, etc.).

The workshops show that even without specific training in 3D modeling, designers were able to import models from databases and put them in a virtual environment created from scratch. Adding behavior to this environment on the other hand turned out to be difficult even for experienced prototypers. While it was expected that prototypers would be able to use a generic Game Engine for this, it was found that the learning curve of these tools (in this case the Blender Game Engine) are quite steep. In addition to the steep learning curve it should be considered that the tool will not be used on a daily basis, and that not every design department has a dedicated prototyper (or designers with similar skills) available. This bottleneck could be addressed, either by providing designers with easy to use programming tools for creating interactive prototypes, or by outsourcing this task to experts such as dedicated virtual prototypers (within or outside the design department).

The Tool Selection phase of the case study allowed designers to compose their own tool chain based on experienced gained during the prototype evaluations. Out of the three tool chain components, two are supported by tools that are sufficiently usable and effective, and integrate well with existing tools and databases.

\section{E. Generalization}

Up to this point, the development of the application prototype and the selection of tools have been company specific activities, leading to an application of VR for early stage design tasks for this particular company. In the final stage of the case study we investigated how well the results translate to other companies and design domains; can other companies benefit from a similar application, and if so, do they have different requirements regarding tools? During a group meeting attended by three design companies (a product design agency, a truck design multinational and a machine design multinational), designers were asked to find an analogue of the Virtual Printshop application that is relevant in their own practice, and subsequently indicate how well the accompanying tools integrate with their existing tool chain (see figure 7).

1) Generalization of the Application: After demonstrating the virtual printshop to the session participants, the designers were asked to break down the application into a generic "virtual context' (e.g. the printshop) and generic 'virtual objects' (e.g. printers, furniture, avatars, etc.). These generic elements were given a new and concrete shape by the designers, for instance by letting the virtual context become a highway and the virtual objects trucks and cars. In addition to describing their virtual environments the participants were also asked to compare their envisioned applications to the pre-defined 
application and indicate if for instance the level of realism or level of complexity should be above or below the level presented in the case study (i.e. use it as a benchmark).

Two of the three companies were able to identify applications analogue to the virtual printshop in their own design practice.

1) Virtual Bakery Shop - The design agency ( $A$ in figure 7) selected one specific product for this session that suits the VR application presented. The selected product is a machine that bakes/finishes bread inside a shop or supermarket. A VR application similar to the Virtual Printshop could be a time saving application in their design process by supporting the communication with their customers. The designers envision a virtual bakery shop, in which their product concept as well as the current machines, objects and people present in the bakery would be represented. Aspects such as safety, product routing and product presentation could be incorporated in the application.

2) Virtual Factory - The machine designers ( $B$ in figure 7) envision a fairly straight-forward translation of the original application. They would use a 'Virtual Factory' to show a client (the buyer of a new machine) a realistic representation of the proposed solution. This application would primarily support sales and negotiation phases, but in a way also provide validation of assumptions and design proposals; the client will be able to indicate whether or not a proposed solution meets the requirements. Compared to the original virtual printshop however, the primary aim would not be to evaluate or improve design solutions.

The truck design company ( $C$ in figure 7 ) was unable to describe an analogue application. The only translation would be to use the truck cabin as a virtual context, in which truck drivers can look around and for instance experience future dashboard or cabin concepts. However, this application was not considered very relevant by the company.

2) Generalization of Tools: After identifying analogue applications, the companies were introduced to the tool chain used in the case study to realize the applications and asked to discuss the compatibility of the tool chain with their current tool chains. As shown in figure 7, it was generally agreed that Geometry Modeling is quite well supported by tools currently in use (usually the company's CAD software or model database) and that Scene Integration can be either covered by current CAD software or supported by the tool presented in the case study. Behaviour Modeling is more difficult to integrate with the tools and skills of design departments, as it is not considered a core task of early stage product design. Consequently, nor the people or the tasks are generally available to do Behaviour Modeling. Figure 7 shows that company B 'solves' this by simply leaving out the Behaviour Modeling step; it was argued that even without having an interactive environment, the application would be beneficial. Company A on the other hand indicated that the required tools and skills would be acquired externally rather than leaving out this part of the tool chain altogether.

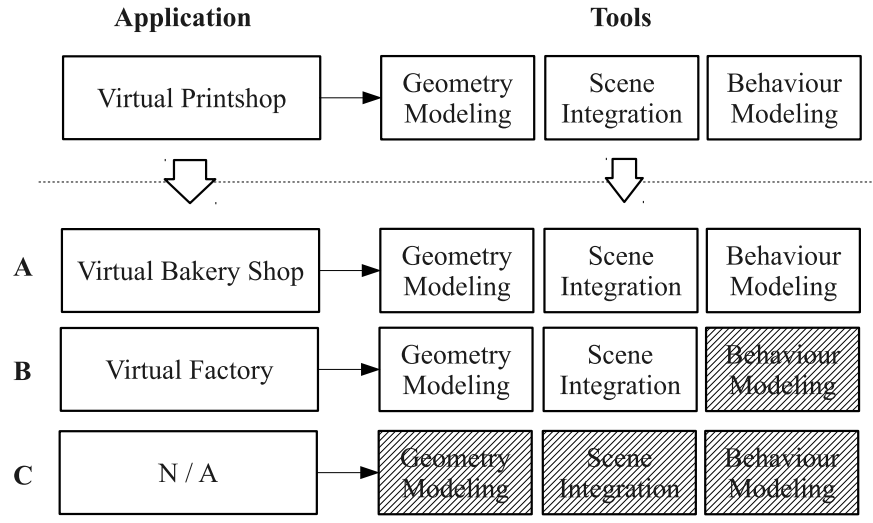

Fig. 7. Three additional companies were involved to generalize the case study results, including a product design agency (A), a machine design multinational (B) and a truck design multinational (C). Etched areas depict tool chain components that were not included in the tool chain of the particular company.

\section{DISCUSSION}

The Virtual Printshop that was developed in the case study does not yet provide all the functions required for practical use (for instance, the application does not support importing models from external sources). The current proof of concept allowed for a qualitative assessment of effectiveness of the application, but was not sufficiently polished to be used by actual end-users and skew quantitative data. The discussion presented in this section therefore focuses on the experiences and insights gained while carrying out the case study.

The approach as implemented in the presented case study has been successful in creating awareness about VR among design practitioners, and in exploring and refining opportunities for effective applications. The close collaboration with designers ensured that the application is useful in practice; designers constantly indicated whether or not they would 'see this work' in real-life. For example, after presenting a storyboard that illustrated potential applications of augmented reality, a discussion was triggered about practical issues; "do we send an AR kit to our customers, or do we invite them over to do it here?". Interestingly, the discussion did not focus on technical arguments to make this decision, but rather practical ones (e.g. "a customer may not understand how to use the $A R$ kit" or "if customers augment their own use context, they'll have a realistic experience and it will save us time of modeling use contexts ourselves"). Discussions like this not only provide the researcher with a better understanding of practical requirements of VR applications, but also indicate that the participating designers understand the technologies well enough to engage in discussions about it. Another positive side-effect of the close collaboration is that the design department becomes (and remains) committed to participate in the case study; they were part of creating the initial application and like to stay involved in its further development, evaluation and validation. Moreover, a proper understanding of the envisioned application seems to reduce the threshold for designers to start using or learning to use new tools; they are willing to invest time and effort if they are aware of the benefits gained in return. 
A downside of close collaboration with practitioners that was encountered during the case study is the infinite number of 'new opportunities' that emerges while discussing the application. This issue is difficult to handle because the researcher, who in the end is in charge of the development, needs to decide whether or not a new opportunity should be taken into account. Some of the opportunities are low hanging fruits, meaning that the application is improved or extended without significant development effort. For example, designers indicated that the virtual printshop could also be used to discuss and communicate room layouts and related issues, such as the impact of the layout on total costs, the environmental impact or maintenance. While this use of the virtual printshop is different from the originally envisioned application, it does illustrate its versatility which in turn can help the adoption of the application within the company. Other opportunities and ideas proposed during discussions are less easy to implement, and do not always contribute to the application. It frequently occurred that designers proposed to use technologies (e.g. motion tracking suits or 3D displays) without motivating why they would want to use it. In these cases it is important for the researcher (or in general; the facilitator of these discussions) to assess the usefulness of adding technologies.

\section{CONCLUSION}

In this paper we addressed the gap between the potential benefits of VR applications in early stages of a user centered PDP and the tools available for designers to realize these applications. In the presented case study we identified a useful application of VR for the participating design department, and provided a selection of tools that allows designers to realize the application themselves. While the resulting Virtual Printshop is a relatively low-end form of VR it provides an effective facilitating role in early stage design activities. The test case showed that reviewing and acting out workflows in the Virtual Printshop is considered a valuable addition to existing methods, mainly because the virtual environment provides a realistic and familiar use context. Acting out the workflow in these contexts triggers participants to express knowledge and feedback that might otherwise be left out. A generalization of the cases study results showed that the application can be translated to several other design domains.

With respect to tools, similarities were found in how different design domains integrate $V R$ design tools with their existing tool chains. Designers prefer to import 3D models from existing repositories rather than modeling everything themselves, even if this results in more accurate models. Gathering models and integrating this in a virtual environment is considered a feasible task for design departments, either with existing tools or with tools available elsewhere on the market. Behavior modeling (e.g. programming the 3D models and environments) is considered a difficult skill that is not always available within design departments. Given the low use frequency of the tool and required investment in time and money, training designers to do this themselves is not always desirable. Alternatively, more user friendly (lower threshold) tools are to be found or created to cover this part of the tool chain.

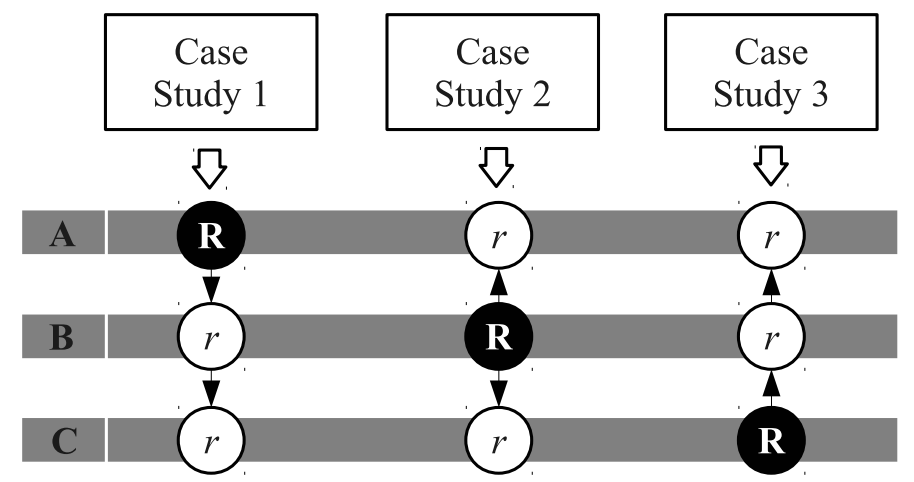

Fig. 8. The results of two additional case studies $(R)$ are translated to results (r) relevant for industrial partners A, B and C.

\section{A. Future Work}

The Virtual Printshop originally aimed to facilitate communication between designers and end-users. The test sessions in the case study however only involved product designers, mainly because of the experimental status of the VR applications. In follow-up projects, the company continued working with some of the tools (in this case a combination of SweetHome3D and Google 3D Warehouse) to improve communication with clients. These follow-ups hopefully lead to opportunities to further evaluate the Virtual Printshop with actual end-users.

The presented case study is the first in a series of three industrial case studies in which the approach is implemented. Each case study features a different industrial partner, allowing for a comparison of the individual case study results as shown in figure 8 . The resulting $3 \times 3$ matrix provides the content of a more elaborate framework on how to use VR to facilitate user centered design activities in the early stages of a PDP. Furthermore, the case studies allow us to iteratively improve the approach presented in this paper, leading to a more founded method for identifying useful and usable VR applications in the early stages of a user centered design process.

\section{ACKNOWLEDGMENT}

The authors gratefully acknowledge the support of the Innovation-Oriented Research Programme 'Integral Product Creation and Realization (IOP IPCR)' of the Netherlands Ministry of Economic Affairs, Agriculture and Innovation. We would also like to thank everyone who participated in the case study.

\section{REFERENCES}

[1] Google 3D warehouse. http://sketchup.google.com/3dwarehouse/, October 2012 .

[2] Sweet home 3D. http://www.sweethome3d.com/index.jsp, October 2012.

[3] Ernesto G Arias and Gerhard Fischer. Boundary objects: Their role in articulating the task at hand and making information relevant to it. 2000.

[4] Selim Balcisoy, Marcelo Kallmann, Pascal Fua, and Daniel Thalmann. A framework for rapid evaluation of prototypes with augmented reality. pages 61-66, Seoul, Korea, 2000. ACM.

[5] Allen Bierbaum, Christopher Just, Patrick Hartling, Kevin Meinert, Albert Baker, and Carolina Cruz-Neira. ACM SIGGRAPH ASIA 2008 courses. pages 1-8, Singapore, 2008. ACM.

[6] Blender. Free and open source 3D modeling, animation and game engine software. http://www.blender.org, July 2011. 
[7] S. V.G Cobb, M. D D'Cruz, and J. R Wilson. Integrated manufacture: A role for virtual reality? International Journal of Industrial Ergonomics, 16(4-6):411425, 1995.

[8] Kim Halskov and Peter Dalsgrd. Inspiration card workshops. In Proceedings of the 6th conference on Designing Interactive systems, pages 2-11, University Park, PA, USA, 2006. ACM.

[9] C. Herstatt and B. Verworn. The Fuzzy front end of innovation. Bringing Technology and Innovation into the Boardroom, Houndmills and New York: Palgrave MacMillan, page 347373, 2004.

[10] Kato Hirokazu. ARToolKit: library for vision-based augmented reality. IEIC Technical Report (Institute of Electronics, Information and Communication Engineers), 101(652(PRMU2001 222-232)):79-86, 2002.

[11] A. Jimeno and A. Puerta. State of the art of the virtual reality applied to design and manufacturing processes. The International Journal of Advanced Manufacturing Technology, 33(9):866874, 2007.

[12] M. A. Kaulio. Customer, consumer and user involvement in product development: A framework and a review of selected methods. Total Quality Management, 9(1):141, 1998.

[13] S. Kujala1. Effective user involvement in product development by improving the analysis of user needs. Behaviour \& Information Technology, 27(6):457-473, 2008.

[14] K. Kuutti, K. Battarbee, S. Sde, T. Mattelmki, T. Keinonen, T. Teirikko, and A. Tornberg. Virtual prototypes in usability testing. In Hawaii International Conference on System Sciences, volume 5, page 5029, Los Alamitos, CA, USA, 2001. IEEE Computer Society.

[15] Blair MacIntyre, Maribeth Gandy, Steven Dow, and Jay David Bolter. DART: a toolkit for rapid design exploration of augmented reality experiences. In Proceedings of the 17th annual ACM symposium on User interface software and technology, UIST '04, page 197206, Santa Fe, NM, USA, 2004. ACM. ACM ID: 1029669.

[16] Dennis Nobelius and Lars Trygg. Stop chasing the front end process management of the early phases in product development projects. International Journal of Project Management, 20(5):331-340, July 2002.

[17] Stig Ottosson. Qualified product concept design needs a proper combination of pencil-aided design and model-aided design before product data management. Journal of Engineering Design, 9(2):107, 1998.

[18] Gerhard Reitmayr and Dieter Schmalstieg. An open software architecture for virtual reality interaction. In Proceedings of the ACM symposium on Virtual reality software and technology, pages 47-54, Baniff, Alberta, Canada, 2001. ACM.

[19] J.P. Thalen and MC van der Voort. User centred methods for gathering VR design tool requirements. In Joint Virtual Reality Conference of EGVE-EuroVR, page 7581, 2011.

[20] Martijn Tideman, Mascha van der Voort, and Fred van Houten. A new product design method based on virtual reality, gaming and scenarios. International Journal on Interactive Design and Manufacturing, 2(4):195-205, October 2008.

[21] Tore Urnes, smund Weltzien, Anne Zanussi, Solveig Engbakk, and Julie Kleppen Rafn. Pivots and structured play: stimulating creative user input in concept development. In Proceedings of the second Nordic conference on Human-computer interaction, pages 187-196, Aarhus, Denmark, 2002. ACM.

[22] G. N Vavoula, M. Sharples, and P. D Rudman. Developing the'Future technology workshop'method. In Proceedings of the International Workshop on Interaction Design and Children, Aug, page 2829, 2002.

[23] Y. Wang, T. Langlotz, M. Billinghurst, and T. Bell. An authoring tool for mobile phone AR environments. In Proceedings of New Zealand Computer Science Research Student Conference, volume 9, page 14, 2009.

[24] D. Weidlich, L. Cser, T. Polzin, D. Cristiano, and H. Zickner. Virtual reality approaches for immersive design. International Journal on Interactive Design and Manufacturing (IJIDeM), 3(2):103-108, March 2009.

[25] T. E. Wright and G. Madey. A survey of technologies for building collaborative virtual environments. The International Journal of Virtual Reality, 8(1):5366, 2009. 